\title{
Lattice dynamics of single-walled carbon nanotubes
}

\author{
V. N. Popov* and V. E. Van Doren \\ University of Antwerpen (RUCA), Groenenborgerlaan 171, B-2020 Antwerpen, Belgium \\ M. Balkanski \\ Université Pierre et Marie Curie, 4 Place Jussieu, F-75252 Paris Cedex 05, France
}

(Received 14 May 1998)

\begin{abstract}
In this paper, the theory of lattice dynamics of single-walled carbon nanotubes is presented. The screw symmetry of the system is used to reduce the rank of the dynamical matrix to six, independent of the number of atoms in the unit cell. Calculations of the lattice dynamics are carried out within a valence force field model and of the Raman intensity - within a bond-polarizability model. It is found that the breathing mode frequency is inversely proportional to the radius of the tube that can be used for the characterization of the samples on the basis of Raman-scattering data. The results for the Raman intensity are compared to available Raman spectra. [S0163-1829(98)06434-0]
\end{abstract}

The carbon multiwalled nanotubes were first discovered as a by product of the fullerene production in an electric arc between two graphite rods. ${ }^{1}$ Introducing transition metals in the graphite electrodes of the electric arc, it was made possible to produce single-walled nanotubes (further on, called just "nanotubes", or "tubes"). ${ }^{2}$ Recently, a high yield of nanotubes, forming bundles of parallel tubes ("ropes"), has been obtained by laser ablation of a graphite target. ${ }^{3}$ In this case, the X-ray diffraction (XRD) measurements yielded $13.8 \pm 0.2 \AA$ for the mean diameter of the tubes. Transmission electron microscope (TEM) probes of similar samples 4 revealed that the tube diameter distribution was consistent with the range of armchair tubes $(8,8),(9,9),(10,10)$, and $(11,11)$. Later, scanning tunneling microscope (STM) studies on ropes of nanotubes, synthesized by the same group, showed that nanotubes with a wide range of chiral angles were present. ${ }^{5,6}$

In view of the possible technological applications of the nanotubes, it is therefore of primary interest to make a precise characterization of the synthesized samples. Since the direct measurement of the structural parameters of the nanotubes is a rather time consuming and expensive procedure, it is highly desirable to use, for this purpose, alternative methods such as Raman-scattering spectroscopy. ${ }^{4}$

In this work the theory of the lattice dynamics of nanotubes is developed. The screw symmetry of the system is used to reduce the size of the dynamical matrix to six for all tube types, which allows one to study the vibrations of arbitrary nanotubes and, more generally, on any helical structure. In this respect the model differs from the zone-folding (ZF) method. ${ }^{7}$ The calculations of the phonons are accomplished within a valence force field (VFF) model with model parameters taken over from graphite. The Raman intensity is estimated using a bond-polarizability model. ${ }^{8}$ The breathing mode frequency is found to be inversely proportional to the tube radius, which can be helpful for the characterization of nanotube samples on the basis of Raman-scattering data.

Following Refs. 9-11, a nanotube can be viewed as constructed by rolling up a graphene sheet into a seamless cylinder leading to the coincidence of the lattice point $O$ at the origin and another one $O^{\prime}$ with indices $\mathbf{L}=\left(L_{1}, L_{2}\right)$. A tube is uniquely specified by the pair of integers $\left(L_{1}, L_{2}\right)$ or by its radius $R$ and chiral angle $\theta$. The latter is defined as the angle between the vector $\overrightarrow{O O^{\prime}}$ on the sheet and the nearest zigzag of carbon-carbon bonds with values in the interval $0 \leqslant \theta$ $\leqslant \pi / 6$. The tubes are called achiral for $\theta=0$ (" zigzag", type) and $\theta=\pi / 6$ ("'armchair'" type), and chiral for $\theta \neq 0, \pi / 6$.

All the atoms of a tube can be reproduced by the use of an atomic pair and two different screw operators ${ }^{12}$ (see Fig. 1). A screw operator $\{S \mid \mathbf{t}\}$ rotates a position vector of an atom at an angle $\phi$ about the tube axis and translates it a vector $\mathbf{t}$ along the same axis. Thus, the equilibrium position vector $\mathbf{R}(\mathbf{l} k)$ of the $k$ th atom of the lth pair of the tube is obtained from $\mathbf{R}(k) \equiv \mathbf{R}(\mathbf{0} k)$ using two screw operators $\left\{S_{1} \mid \mathbf{t}_{1}\right\}$ and $\left\{S_{2} \mid \mathbf{t}_{2}\right\}$,

$$
\mathbf{R}(\mathbf{l} k)=\left\{S_{1} \mid \mathbf{t}_{1}\right\}^{l_{1}}\left\{S_{2} \mid \mathbf{t}_{2}\right\}^{l_{2}} \mathbf{R}(k)=S_{1}^{l_{1}} S_{2}^{l_{2}} \mathbf{R}(k)+l_{1} \mathbf{t}_{1}+l_{2} \mathbf{t}_{2} .
$$

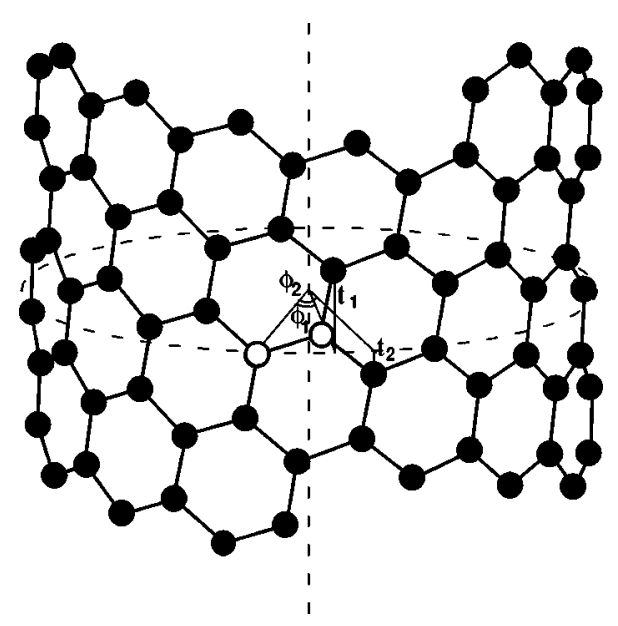

FIG. 1. A front view of a nanotube. The closed dashed line is the circumference and the straight dashed line is the axis of the tube. The nanotube can be constructed by mapping the two atoms of the unit cell (depicted by empty points) unto the entire cylindrical surface using two different screw operators. 
Here $\mathbf{I}=\left(l_{1}, l_{2}\right), l_{1}$ and $l_{2}$ are integers labeling the atomic pair, and $k=1,2$ enumerates the atoms in the pair. It is convenient to adopt the notation $S(\mathbf{l})=S_{1}^{l_{1}} S_{2}^{l_{2}}$ and $\mathbf{t}(\mathbf{l})=l_{1} \mathbf{t}_{1}+l_{2} \mathbf{t}_{2}$, and to rewrite Eq. (1) as

$$
\mathbf{R}(\mathbf{l} k)=S(\mathbf{l}) \mathbf{R}(k)+\mathbf{t}(\mathbf{I}) .
$$

For small displacements $\mathbf{u}(\mathbf{l} k)$ of the atoms from their eqiulibrium positions, the harmonic approximation may be used for the potential energy of the tube and the resulting equations of motion are derived in the form

$$
m_{k} \ddot{u}_{\alpha}(\mathbf{l} k)=-\sum_{\mathbf{l}^{\prime}, k^{\prime}, \beta} \Phi_{\alpha \beta}\left(\mathbf{l} k, \mathbf{l}^{\prime} k^{\prime}\right) u_{\beta}\left(\mathbf{l}^{\prime} k^{\prime}\right) .
$$

Here, $\Phi_{\alpha \beta}\left(\mathbf{l} k, \mathbf{l}^{\prime} k^{\prime}\right)$ are the force constants.

The helical symmetry of the nanotube suggests that we search for solutions to Eq. (3) of the type

$$
u_{\alpha}(\mathbf{l} k)=\frac{1}{\sqrt{m_{k}}} \sum_{\beta} S_{\alpha \beta}(\mathbf{l}) e_{\beta}(k \mid \mathbf{q}) \exp [i \mathbf{q} \cdot \mathbf{l}-i \omega(\mathbf{q}) t],
$$

representing a wave with wave vector $\mathbf{q}=\left(q_{1}, q_{2}\right),-\pi$ $\leqslant q_{1}, q_{2} \leqslant \pi$, and angular frequency $\omega(\mathbf{q})$.

Substituting Eq. (4) in Eq. (3), we get a system of linear equations that can be cast in the form

$$
\omega^{2}(\mathbf{q}) e_{\alpha}(k \mid \mathbf{q})=\sum_{k^{\prime}, \beta} D_{\alpha \beta}\left(k k^{\prime} \mid \mathbf{q}\right) e_{\beta}\left(k^{\prime} \mid \mathbf{q}\right),
$$

where the dynamical matrix is defined as

$$
D_{\alpha \beta}\left(k k^{\prime} \mid \mathbf{q}\right)=\frac{1}{\sqrt{m_{k} m_{k^{\prime}} \mathbf{I}^{\prime}, \gamma}} \sum_{\alpha \gamma} \Phi_{\alpha}\left(\mathbf{0} k, \mathbf{l}^{\prime} k^{\prime}\right) S_{\gamma \beta}\left(\mathbf{l}^{\prime}\right) \exp \left(i \mathbf{q} \cdot \mathbf{l}^{\prime}\right) .
$$

The eigenfrequencies $\omega(\mathbf{q})$ are solutions of the characteristic equation

$$
\left\|D_{\alpha \beta}\left(k k^{\prime} \mid \mathbf{q}\right)-\omega^{2}(\mathbf{q}) \delta_{\alpha \beta} \delta_{k k^{\prime}}\right\|=0 .
$$

Using the eigenvalues $\omega^{2}(\mathbf{q} j)$, one can obtain from Eq. (5) the corresponding eigenvectors $e_{\alpha}(k \mid \mathbf{q} j)(j=1,2, \ldots, 6)$. From Eq. (6) it can be proven that $\mathbf{D}(\mathbf{q})$ is Hermitian and, therefore, $\omega^{2}(\mathbf{q} j)$ are real and $e_{\alpha}(k \mid \mathbf{q} j)$ may be chosen orthonormal.

The rotational boundary condition $\mathbf{u}(\mathbf{l}+\mathbf{L}, k)=\mathbf{u}(\mathbf{l} k)$ imposes the constraint $q_{1} L_{1}+q_{2} L_{2}=2 \pi m$ ( $m$ is an integer) on the q-vector components. ${ }^{9-12}$ The theory presented above is valid for any helical structure and no translational periodicity was accounted for so far. It can be shown ${ }^{10,11}$ that in all nanotubes such symmetry exists and the primitive translation vector of a given tube connects the lattice point at the origin with another one with indices $\mathbf{N}=\left(N_{1}, N_{2}\right)$ given by

$$
N_{1}=\frac{L_{1}+2 L_{2}}{d}, \quad N_{2}=-\frac{2 L_{1}+L_{2}}{d} .
$$

Here $d$ is equal to the highest common divisor $d^{\prime}$ of $L_{1}$ and $L_{2}$ if $L_{1}-L_{2}$ is not a multiple of $3 d^{\prime}$ or $d$ is equal to $3 d^{\prime}$ if $L_{1}-L_{2}$ is a multiple of $3 d^{\prime}$. The total number $n$ of atomic pairs in the unit cell is

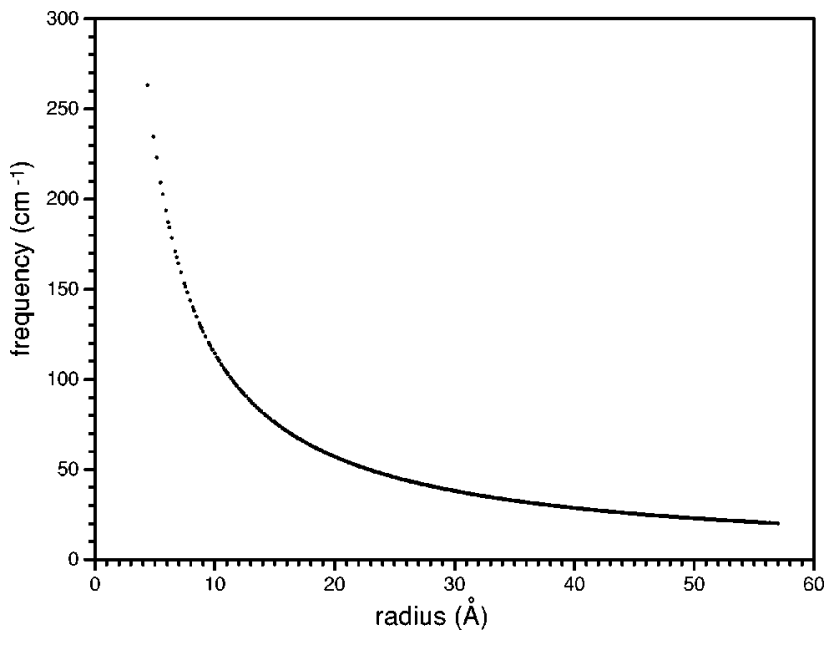

FIG. 2. The frequency of the breathing mode as a function of the radius of the nanotube.

$$
n=\frac{2\left(L_{1}^{2}+L_{2}^{2}+L_{1} L_{2}\right)}{d} .
$$

The translational periodicity of a tube leads to a Bloch type of displacement field with a one-dimensional wave vector $q$,

$$
q=q_{1} N_{1}+q_{2} N_{2} .
$$

It can be shown that for each $q$ there are $6 n$ eigenvectors but lesser eigenvalues due to the degeneracy of some of the modes.

The force constants are invariant under infinitesimal translations along and perpendicular to the tube axis that leads to the translational sum rules and to three zone-center zero-frequency modes. The infinitesimal rotational invariance condition imposed on the force constants gives rise to a rotational sum rule and to an additional zero-frequency mode.

The theory of the lattice dynamics of carbon nanotubes can be used now to calculate the phonon dispersion curves for arbitrary $q$ within a phenomenological model. The experimental measurements do not provide enough data on the structural parameters of the tubes such as bond lengths and bond angles, but the results of $a b$ initio calculations of the optimized structures of nanotubes show that these quantities differ only by a few percent with those in graphite and that the carbon atoms do not lie on cylindrical surfaces. ${ }^{13}$ However, it is found here that such minor differences do not much affect the eigenmodes. Therefore, in the calculations presented in this paper, it was accepted that for a given tube (1) all bond lengths are equal to $1.42 \AA$, the same as those in the graphene sheet, (2) all bond angles are equal but, of course, less than $120^{\circ}$, and (3) all atoms lie on a cylindrical surface. The calculation of the eigenmodes in nanotubes is carried out here within the VFF model with nearest-neighbor stretch, next-to-nearest neighbor stretch, in-plane bend, outof-plane bend, and twist interactions. ${ }^{14}$ The results for the phonon dispersion curves will be given in detail elsewhere. From them it follows, in particular, that the frequency $\omega_{b}$ of the zone-center in-phase radial ("breathing") mode is inversely proportional to the radius $R$ of the tube (Fig. 2) and that in a wide region, $4 \AA<R<55 \AA$, it can be approxi- 
mated by $1147.81 / R^{1.00181}$ (in $\mathrm{cm}^{-1}$ ). However, $\omega_{b}$ is not found to be dependent on the chiral angle. The Raman spectra calculated in this paper exhibit a peak of high intensity due to the breathing mode that dominates the low-frequency region of the spectra. Therefore, the $R$ dependence of $\omega_{b}$ can be used for direct determination of the average radius of nanotubes on the basis of Raman data only. The $1 / R$ dependence of $\omega_{b}$ can also be deduced from a simple forceconstant model. Let us consider a zigzag monoatomic chain with bond lengths $a$ and bond angles of $120^{\circ}$, which is bent in a cylinder with radius $R$, and let us assume only bondstretching interactions with force constant $\kappa$. For simultaneous radial displacement of all atoms with a distance $\Delta R$, the restoring force acting on an atom is given by $\left(3 \kappa a^{2} / 2 R^{2}\right) \Delta R$. The frequency of the breathing motion of the chain can be found from this expression to be $(3 \kappa / 2 m)^{1 / 2} a / R \propto 1 / R \quad(m$ is the atomic mass) that reproduces the VFF results within a few percent if in both cases one uses the value $\kappa=3.44 \mathrm{mdyn} / \AA$ taken from Ref. 14 .

Recently, resonant Raman-scattering spectra of nanotubes have been obtained. ${ }^{4}$ The TEM and XRD measurements on the samples revealed that the tubes were exclusively of armchair type and it was estimated that only types $(8,8),(9,9)$, $(10,10)$, and $(11,11)$ were consistent with the measured diameter distribution. In these experimental Raman spectra only two strong bands have been observed. The highfrequency doublet at $1567-1593 \mathrm{~cm}^{-1}$ was ascribed to carbon-carbon bond stretching motions and the lowfrequency one, at about $170 \mathrm{~cm}^{-1}$, to breathing motions of the tubes. The assignment of the separate peaks in both bands was made using the results of a ZF model of the lattice dynamics. ${ }^{4}$ While an overall fair agreement was achieved between the theoretical estimations and the Raman data, it has to be noted that the peak at $1567 \mathrm{~cm}^{-1}$ does not correspond in frequency to any predicted zone-center phonon. In an attempt to explain the appearance of this peak, we calculate the Raman intensity for a parallel scattering configuration of a sample of randomly oriented tubes of armchair type, adopting one and the same full width at half maximum of $6 \mathrm{~cm}^{-1}$ for all Raman lines. In the case of tube types from $(8,8)$ to $(11,11)$, the high-frequency region of the obtained spectrum consists of a single broad peak centered at $1593 \mathrm{~cm}^{-1}$, and its shape does not change qualitatively if armchair tubes of larger diameters are also accounted for. When tube types $(5,5),(6,6)$, and $(7,7)$ are included in the calculations, additional steps appear in the spectrum at about 1567, 1578, and $1584 \mathrm{~cm}^{-1}$, correspondingly. Furthermore, a qualitative agreement with the experimental spectra in Ref. 4 is reached if tubes of types $(8,8)-(11,11)$ with a Gaussian weight $\exp \left[-\left(L_{1}-9.5\right)^{2}\right]$ are taken into account together with the same total number of $(5,5)$ tubes [see Fig. 3(a)]. The latter have the same diameter as the $\mathrm{C}_{60}$ molecules and are found to be of high stability. Thus, they are likely to be always present in the samples irrespective of the method of synthesis. Finally, if all tube types are included with the same weight function, the high-frequency doublet is partially smeared out [Fig. 3(b)] On the basis of the high-frequency region of the calculated spectra it can be concluded that even if tubes of other types are also present in the samples, as was suggested in recent STM measurements, ${ }^{5,6}$ their concentra-

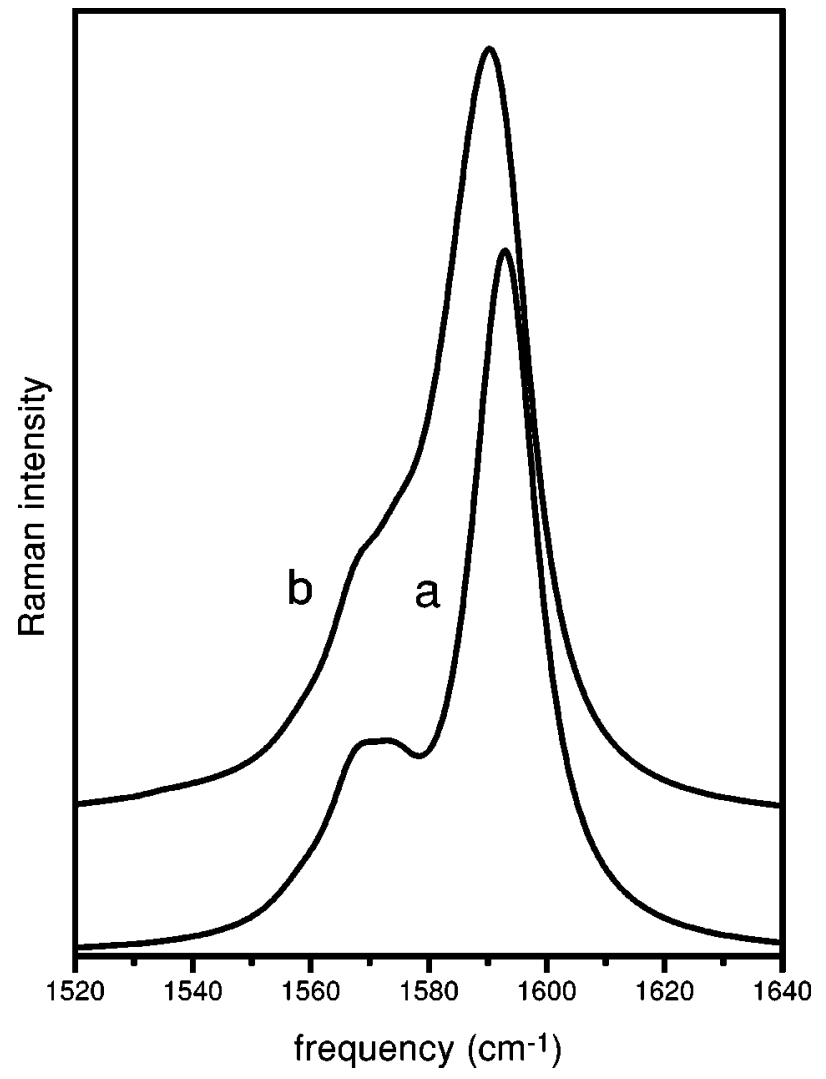

FIG. 3. Calculated Raman spectrum in the region 1520-1640 $\mathrm{cm}^{-1}$ for parallel scattering configuration and for a sample consisting of randomly oriented tubes of (a) armchair type and (b) all tube types with weight factors as described in the text.

tion must be very low. It is clear that additional precise Raman measurements are needed in order to check the validity of the model predictions and to derive the distribution of the tube types in a given sample by fitting the calculated spectra to the measured ones.

The five Raman spectra in Fig. 3 of Ref. 4 show major differences mainly in the low-frequency region. The spectrum measured at the off-resonance excitation with wavelength $\lambda=1320 \mathrm{~nm}$ exhibits only the band at $1600 \mathrm{~cm}^{-1}$. The one at $\lambda=780 \mathrm{~nm}$ is not clearly a resonant one because the peak positions in the band at about $170 \mathrm{~cm}^{-1}$ correspond to the theoretical predictions and to the tube types distribution in the sample (see Table 1 in Ref. 4). In the other three spectra measured at $\lambda=1064,647.1$, and $514.5 \mathrm{~nm}$, the intensity and the position of the low-frequency peaks change with $\lambda$, which is evidence for the resonant character of the scattering. The reason for the appearance of resonant scattering is the closeness of the laser photon energy to the energy difference between spikes in the electron density of states. A comparison of the laser photon energy with the measured $^{5,6}$ and with the calculated ${ }^{15}$ gaps in the electronic density of states (DOS) yields that $\lambda$ $=1064 \mathrm{~nm}(1.17 \mathrm{eV})$ corresponds to the secondary gap in semiconducting tubes, $\lambda=647.1 \mathrm{~nm}(1.92 \mathrm{eV})$ to the primary gap in metallic tubes, and $\lambda=514.5 \mathrm{~nm}(2.41 \mathrm{eV})$ to the secondary gap in metallic tubes. This conclusion, however, does not support the results of previous calculations of the electronic DOS. ${ }^{7}$

Just before submitting this paper for publication, calcula- 
tions of the Raman intensity using a bond-polarizability model were reported. ${ }^{16}$ These authors calculated the phonon dispersions within a force-constant model but the screw symmetry of the tubes was not included explicitly. Instead, the force-constants matrix was deduced by bond rotations and by making additional corrections of the various force constants in order to satisfy the rotational acoustic sum. A $R$ dependence of $\omega_{b}$, but for the range from 3 to $7 \AA$, similar to the one shown here, was also derived. Additionally, the Raman intensity for several tube types, as well as averaged ones over the orientation of the sample, was also calculated.

In conclusion, in this work the lattice dynamics of nanotubes is presented. Essential simplification of the theory is obtained using the screw symmetry of the system. The results of the calculations of the Raman intensity of a nanotube sample within a purely phenomenological approach are used to explain the appearence of the high-frequency doublet observed in the Raman spectra. The dependence of frequency of the breathing mode on the tube radius can be applied to characterize the synthesized samples.

This work was supported partly under Grant No. G2131.94 of the Flemish Science Foundation, partly by the Concerted Action of the University of Antwerp GOABOF-UA Nr.23, and partly under Grant No. F-423 of the Bulgarian National Science Fund. The authors would like to thank C. T. White and J. W. Mintmire (NRL, Washington, DC) for useful discussions and for communicating their work to us prior to publication.
*Permanent address: Faculty of Physics, University of Sofia, BG1164 Sofia, Bulgaria.

${ }^{1}$ S. Iijima, Nature (London) 354, 56 (1991).

${ }^{2}$ S. Iijima and T. Ichihashi, Nature (London) 363, 603 (1993); D. S. Bethune et al., ibid. 363, 605 (1993).

${ }^{3}$ A. Thess et al., Science 273, 483 (1996); J. E. Fisher et al., Phys. Rev. B 55, R4921 (1997).

${ }^{4}$ A. M. Rao et al., Science 275, 187 (1997).

${ }^{5}$ J. W. G. Wildöer et al., Nature (London) 391, 59 (1998).

${ }^{6}$ T. W. Odom et al., Nature (London) 391, 62 (1998).

${ }^{7}$ M. S. Dresselhaus, G. Dresselhaus, and P. C. Eklund, Science of
Fullerenes and Carbon Nanotubes (Academic, New York, 1996).

${ }^{8}$ S. Guha et al., Phys. Rev. B 53, 13106 (1996).

${ }^{9}$ N. Hamada et al., Phys. Rev. Lett. 68, 1579 (1992).

${ }^{10}$ D. H. Robertson et al., Phys. Rev. B 45, 12592 (1992).

${ }^{11}$ R. Saito et al., Phys. Rev. B 46, 1804 (1992).

${ }^{12}$ C. T. White et al., Phys. Rev. B 47, 5485 (1993).

${ }^{13}$ Jae-Yel Yi and J. Bernholc, Phys. Rev. B 47, 1708 (1993).

${ }^{14}$ S. Siebetritt et al., Phys. Rev. B 55, 7927 (1997).

${ }^{15} \mathrm{C}$. T. White and J. W. Mintmire, Nature (to be published).

${ }^{16}$ R. Saito et al., Phys. Rev. B 57, 4145 (1998). 